\title{
LE TRAVAIL DE PAROLE AVEC LES ENSEIGNANTS : UN REMPART FACE AUX NOUVELLES FORMES DE FATIGUE SUBJECTIVE
}

\author{
Ilaria Pirone (Université Paris 8)* \\ https://orcid.org/0000-0002-1731-5602 \\ Jean-Marie Weber (Université Luxembourg)** \\ https://orcid.org/0000-0001-6043-7720
}

\begin{abstract}
RÉSUMÉ
Les expériences de recherche et de formation universitaire des deux auteurs les ont amenés à constater une nouvelle forme de fatigue diffuse chez les enseignants, qu'ils proposent de penser comme une sorte de symptôme social. Cette proposition les conduit à analyser ce mal-être comme une réponse subjective qui donne à lire une écriture à deux du lien social : R.I., Réel, Imaginaire. La lecture de ces expressions de «mal-être social » oblige la psychanalyse à sortir d'une position anti-éthique de savoir pour continuer à faire de sa pratique un appui permettant au sujet de rester connecté à son désir. C'est la visée que les deux chercheurs donnent au dispositif d'analyse clinique des pratiques professionnelles présenté, et qui a été construit pour permettre une remise en circulation de la parole entre les professionnels des métiers de l'humain, comme un rempart possible face aux risques de mélancolisation du lien éducatif.
\end{abstract}

Mots-clefs: Enseignants. Ethique. Discours. Mélancolisation. Dispositif de formation.

\section{RESUMO}

\section{TRABALHAR COM PROFESSORES: UM BALUARTE CONTRA NOVAS FORMAS DE FADIGA SUBJETIVA}

As experiências de pesquisa e formação acadêmica dos dois autores os levaram a observar uma nova forma de fadiga difusa entre os professores, que eles se propõem a pensar como um certo sintoma social. Esta proposta os leva a analisar este mal-estar como uma resposta subjetiva que dá uma leitura da escrita do vínculo social: R.I., Real, Imaginário. A leitura destas expressões de "mal-estar social" obriga a psicanálise a sair de uma posição antiética de conhecimento para

\footnotetext{
Maître de conférences, departement de Sciences de l'éducation, Université de Paris 8. Psychologue clinicienne, psychanalyste. E-mail: ilaria.pirone@univ-paris8.fr

** Maître de conférences, Institut d'Éducation et Societé (InES), Université Luxembourg. Psychanalyste. E-mail: jean-marie. weber@uni.lu
} 
continuar a fazer de sua prática um suporte que permita ao sujeito permanecer ligado a seu desejo. Este é o objetivo que os dois pesquisadores dão ao dispositivo de análise clínica das práticas profissionais apresentadas, e que foi construído para permitir uma recirculação da palavra entre os profissionais das profissões humanas, como um possível baluarte contra os riscos de "mélancolisation" do vínculo educativo.

Palavras-chave: Professores. Ética. Discurso. Melancolização. Dispositivo de treinamento.

\section{ABSTRACT \\ WORKING WITH TEACHERS: A BULWARK AGAINST NEW FORMS OF SUBJECTIVE TIREDNESS}

The two authors' research and university training experiences have led them to observe a new form of diffuse tiredness among teachers, which they propose to think of as a kind of social symptom. This proposal leads them to analyse this malaise as a subjective response that gives a reading of the writing of the social bond: R.I., Real, Imaginary. The reading of these expressions of "social malaise" obliges psychoanalysis to come out of an unethical position of knowledge in order to continue to make its practice a support allowing the subject to remain connected to his desire. This is the aim that the two researchers give to the device for clinical analysis of professional practices presented, and which has been constructed to allow a re-circulation of the speech (parole) between professionals in the human professions, as a possible bulwark against the risks of "melancholisation" of the educational bond.

Keywords: Teachers. Ethics. Discourse. Melancholization. Training device.

Ce qui distingue le discours du capitalisme est ceci: la Verwerfung, le rejet, le rejet en dehors de tous les champs du symbolique avec ce que j'ai déjà dit que ça a comme conséquence. Le rejet de quoi? De la castration. Tout ordre, tout discours qui s'apparente du capitalisme laisse de côté ce que nous appellerons simplement les choses de l'amour, mes bons amis. (LACAN, 2011, p. 96).

Dans nos pratiques de formation à l'université et dans nos recherches, nous rencontrons souvent des enseignants qui sont dans une recherche presque désespérée de solutions pour faire face aux nombreux défis que leur pose leur métier dans une société qui offre de moins en moins d'appuis pour faire avec le Réel. Ils sont souvent des " résistants », voire des « combattants », qui essayent de garder vivante leur mission principale, transmettre, contre vents et marées. Dernière épreuve, le temps de crise dû au COVID-19. Mais parfois, ou à force, les défis leur paraissent géants, ils aspirent alors à trouver de nouveaux concepts et de nouvelles stratégies. Ceux qui sont déjà en fonction depuis un certain temps arrivent souvent dans nos formations pour se ressourcer et pour sortir de certaines formes de répétition du même. Ils espèrent trouver des réponses dans les formations, notamment en sciences de l'éducation.

Nous sommes alors souvent confrontés à un état de fatigue de certains enseignants, accompagné de plaintes récurrentes qui portent sur le fait qu'ils ne savent pas/plus comment faire, ou encore qu'ils ne peuvent pas faire ce qu'on leur demande. Mais que leur demandet-on aujourd'hui? De savoir adapter la pédagogie à chaque enfant pour une « égalité des chances pour tous », individualiser, différencier, inclure; rendre l'enfant autonome; savoir exer- 
cer son autorité avec bienveillance, apprendre à gérer les émotions, les conflits, le groupe classe; mettre en place une évaluation positive bienveillante; être toujours innovant; coopérer avec les familles, les partenaires de l'école, etc. Entre injonctions morales et injonctions pédagogiques, beaucoup d'enseignants se sentent parfois à bout de souffle.

Capturé par un discours qui prône le tout possible, la performance, le sujet se trouve pris au piège d'un état de frustration permanent, et ne trouve parfois d'autres solutions que de (se) laisser tomber. Il répond alors par une forme d'impuissance, le fait de ne pas pouvoir, lié à une dimension de manque imaginaire, manquer de connaissance pour pouvoir. Ce qui est alors mis hors-jeu par ces nouvelles formes de mal-être, terme qui indique autre chose que le malaise que Freud avait inscrit au cœur même de l'existence, c'est le travail de l'impossible.

En nous inspirant de l'essai captivant du philosophe coréen Byung-Chul Han (2014), La société de la fatigue, nous proposons de penser cet état de fatigue chez les enseignants comme une sorte de symptôme social. Cette proposition nous permet d'aborder deux questions en parallèle. Premièrement le fait que les plaintes répétées de nos collègues enseignants, sont aussi parfois les nôtres en tant qu'enseignants universitaires. La répétition de la plainte reflète les effets des remaniements des discours sur le sujet. Cette position de fatigue, ce « se laisser tomber » est une forme de position subjective qui donne à lire une écriture à deux du lien social: R.I., Réel, Imaginaire, ce que Lacan, le 7 janvier 1972, avait qualifié comme « le rejet en dehors de tous les champs du symbolique » (LACAN, 2011, p. 96). Deuxièmement, en lien avec ce qui précède, ce type de "mal-être social », comme d'autres, oblige la psychanalyse à se pencher sur la réalité sociale et la dimension institutionnelle, pour pouvoir entendre le dire au-delà du dit et permettre ainsi au sujet de rester connecté à son désir, qui est toujours désir de l'Autre. Au fond, c'est une proposition qui renoue politique et éthique, deux bords qui se retrouvent parfois disjoints, et à tort, dans nos pratiques psychanalytiques. Nous soutenons qu'il ne suffit pas de se plaindre d'un discours capitaliste qui tente d'exclure la psychanalyse du champ social. La psychanalyse doit montrer ce qui lui importe : travailler avec l'inconscient qui est « le social », " le politique », comme l'affirmait Lacan. C'est dans ce sens que la prise en compte des questionnements, des désirs et du mal-être chez les enseignants nous porte à repenser nos formations. Il s'agit d'actions, c'est ainsi que les aurait peut-être définies Arendt, des actions pensées pour soutenir le travail de lien, une forme d'engagement éthique et politique qui ne se contente pas de constater son émiettement (BAUMAN, 2018), ni de se plaindre d'une forme de déclin sous couvert d'une position nécessairement à la marge. C'est en ce sens que notre désir de nous engager nous porte à mettre au centre de nos enseignements à l'université la question de l'éthique, d'une pratique de l'éthique que nous avons définie comme une éthique de la rencontre. Nos dispositifs de formation visent à décompléter les discours afin que quelque chose de l'impossible permette de mettre un frein à la machine à performance, un os dans la gueule du crocodile, laissant la place pour un lieu de différAnce (DERRIDA, 1972), lieu vide, à partir duquel la parole puisse à nouveau circuler.

\section{Fatigue et discours}

La plainte répétée de beaucoup d'enseignants tourne autour de plusieurs thèmes: autour de quelque chose qui ne serait plus comme avant, comme une forme d'autorité perdue; un lien perdu avec les parents avec qui ils ne parviennent plus à « bien » communiquer; ou encore une incapacité à " gérer » tous ces enfants différents, et plus précisément certaines catégories d'enfants, comme les «perturbateurs ", les " décrocheurs », ou encore les « autistes » (concernant les « dyslexiques», avec eux ça passe plus facilement, parce que les enseignants disent savoir de quoi il s'agit 
et avoir les bons outils). Plus généralement ce mal-être se résume régulièrement par un sentiment de solitude et d'abandon de la part de l'institution, et une forme nostalgique de perte de sens de leur métier. Ce mal-être donne à voir un rapport au travail qui réduit l'homo faber, l'homme moderne qui œuvre, qui fabrique, déjà aliéné selon Arendt par rapport à l'homme d'action, à celui d'animal laborans, celui qui travaille, qui fatigue, pour survivre. ${ }^{1}$ Cette idée d'œuvrer, et donc de fabriquer et créer par et dans le travail est ce à quoi Freud faisait allusion dans une petite note de Malaise dans la culture:

L'activité professionnelle, quand elle est librement choisie, donne une certaine satisfaction et permet aussi de mettre à profit, en les sublimant, des prédispositions existantes, des élans pulsionnels qui trouvent une prolongation dans l'activité ou s'en trouvent renforcés. Et pourtant, les hommes font peu de cas du travail comme moyen d'accéder au bonheur. (FREUD, 2010, p. 67).

En effet se pose la question suivante: pourquoi certains enseignants ont-ils du mal à se positionner comme des " praticiens réflexifs » qui jouissent de travailler dans un des «métiers de lien » (CIFALI, 1994). Si on suit Freud, cette position risque au fond de décrocher l'investissement professionnel du circuit du désir. Selon notre hypothèse ce phénomène est favorisé par l'accentuation d'un processus de transformation de ces métiers. Le discours scientifique se transformant en discours du maître, met l'enseignant à la place de l'esclave, comme l'expliquait déjà Hegel, et la technisation se teint de moralisation.

Chaque époque pédagogique s'est construite sur des savoirs considérés comme mythologiques, philosophiques ou scientifiques, mais ce qui semble caractériser notre époque pédagogique c'est une forme de discours scientifique à tendance totalitariste, se revendiquant comme unique, et porteur de vérités incontestables,

1 Nous reprenons ici la distinction qu'Arendt fait dans sa définition de la vita activa théorisée dans la Condition de l'homme moderne (ARENDT, 1983). puisque evidence based. Au nom du bien-être de l'enfant, il construit un paradigme neuro-cognitivo-émotionnel (les émotions étant la seule partie «molle » de l'homme acceptée dans ce paradigme), qui doit expliquer comment fabriquer un être performant et facilement adaptable à la réalité, ce qui implique en miroir un professionnel tout aussi performant et capable de s'adapter à toute situation. Ce paradigme se soutient de trois actions qui composent ce que nous avons défini comme le tripode imaginaire contemporain de l'acte éducatif: diagnostiquer, connaître, gérer (PIRONE ; TISSOT, 2020). Il est alors nécessaire de pouvoir connaître toute atypie de l'enfant, de pouvoir repérer, diagnostiquer toute différence. Une fois l'atypie nommée et donc catégorisée, il faut alors connaître tout ce que la science a trouvé de cette atypie, puisque connaître permet de mieux gérer. Au nom du bien de l'enfant, ce tripode imaginaire, diagnostiquer, connaître, gérer, forclos le cadre symbolique du lien éducatif qui se soutient lui des actes de nomination, transmission, humanisation. Le danger est alors l'exclusion. Exclusion des enfants, bien sûr, qui pris dans ces logiques de gestion, où $\mathrm{au}$ fond le modèle d'inclusion scolaire risque de reproduire des écueils du modèle d'intégration, une fois diagnostiqués et rangés dans une catégorie, deviennent invisibles. Mais ce qui nous intéresse pour cette contribution est le risque d'une forme de mélancolisation de la position enseignante, ${ }^{2}$ mettant ainsi en échec la construction du lien éducatif. Confrontés aux multiples apports des sciences de et sur l'éducation - autrefois nommés pédagogie les enseignants risquent d'être pris dans un sentiment d'inadéquation, d'un jamais assez bien, et pourrait-on dire, d'un jamais assez bon face à un Grand Autre fantasmé. Assujetti au seul discours scientifique certains enseignants perdent ainsi de vue l'enjeu sûrement immense et pourtant si simple: être présent à l'enfant pour qu'un lien de transmission soit

2 Nous nous inspirons de la proposition d'Olivier Douville sur la «mélancolisation du lien social » (DOUVILLE, 2001). 
possible, travail d'humanisation. Pouvoir être présent suppose un travail de recherche pour pouvoir créer et trouver un lieu de rencontre avec l'enfant, son altérité, ses questions. Or la fabrique des catégories de plus en plus pointues d'enfants anormaux avec leur cortège de dispositifs pensés pour permettre la "gestion » pédagogique des anomalies, en arrive à des points où les enseignants plus jeunes en termes de carrière n'osent pas penser qu'ils peuvent proposer des choses eux-mêmes et prendre des initiatives. Ils sont tellement gavés de méthodes pendant leur formation, qu'ils risquent de ne pas se sentir autorisés à tout simplement créer face à la surprise de l'inconnu, qui est le principe au cœur de toute relation éducative. Mais si le danger de ce processus de technicisation a été déjà pointé par nombreux chercheurs d'orientation psychanalytique, ce qui a été moins surligné c'est la dimension moralisante qui soutient aussi la traduction pédagogique de ce paradigme scientifique neuro-cognitivo-émotionnel. Des formules comme la " gestion positive des émotions », " école de la bienveillance », parmi d'autres, illustrent comment le discours institutionnel actuel est tout centré sur le « bien » et la « positivité », où tout semble fait pour escamoter le danger d'une possible manifestation du conflit psychique, de l'ambivalence constitutive de tout lien entre êtres humains. Il est le résultat d'une «psychopolitique néolibérale», qui « [...] détruit l'âme humaine, qui n'est rien moins qu'une machine à positiver » (HAN, 2016, p. 46). Ces nouvelles formules qui au nom du bien de l'enfant mettent en permanence l'accent sur le possible, au fond fabriquent des formes écrasantes de culpabilité et tout son lot d'agressivité, difficilement bienveillante. On retrouve ce croisement entre technicisation et moralisation jusqu'à la création de nouvelles catégories d'enfants anormaux comme par exemple celle des « enfants perturbateurs. C'est une étiquette née tout d'abord du néo-langage ordinaire professionnel où l'on a commencé à définir les enfants " agités », comme " perturbateurs », pour ensuite faire un pas de plus et relier le trouble de l'ordre idéalisé à une explication scientifique, les troubles du comportement. L'enfant troubleur de l'ordre a trouvé sa case, l'ordre se trouve imaginairement rétabli. Nous ne sous-estimons pas les difficultés auxquelles sont confrontés les professionnels de l'éducation, mais nous souhaitons pointer le risque d'un tel fonctionnement. Il peut produire des nouvelles formes de désengagement subjectif avec des conséquences importantes dans les processus de transmission, surtout chez les enseignants les plus jeunes qui se tiennent par crainte au strict suivi des programmes dans les formes préconisées par l'institution et n'osent pas transmettre leur savoir. Ils n'osent pas transmettre à partir de leur savoir, entendu aussi comme savoir de la vie, savoir des impossibles et en fin de compte de leur vérité de désir. Ces transformations font signe d'un rapport de plus en plus normatif aux métiers de l'éducation laissant peu de place à sa dimension éthique. Si on reprend le « nœud bo » de Lacan, elles dessinent une forme de nouage (intenable) à deux, Imaginaire-Réel, laissant de côté le registre Symbolique: cela implique que c'est par le registre Imaginaire qu'on essaye de boucher le trou du Réel. C'est cet imaginaire écrasant que nous retrouvons dans ce rapport normatif à l'éducation et dans cette morale de la bienveillance et de la positivité, qui essayant de voiler le Réel par des formes de l'imaginaire, empêche tout travail de liaison. Si dans une classe, les effets de cette écriture peuvent être une incapacité des enseignants à nouer le Réel avec le Symbolique, le pulsionnel avec la parole et la culture, à l'échelle sociale, ces écritures du lien social, nous permettent peut-être de lire ces nouvelles formes de haine déliée qui sont en train de fragiliser nos systèmes démocratiques.

\section{Faire de la place au vide}

Ces réflexions issues de nos recherches et des rencontres avec les professionnels dans 
nos formations ne font que renforcer notre idée de tenir bon dans cette difficile position d'un enseignement universitaire qui se soutient du discours psychanalytique (LACAN, 1978) dans le champ de l'éducation, à condition que nos formations soient un espace de recherche toujours ouvert. Ce choix nous conduit à chercher sans cesse comment faire de nos enseignements ce lieu vide dont parle Lacan. Il s'agit de chercher comment transmettre à partir du principe de division subjective, à partir donc d'une dimension de décomplétude qui est le propre du savoir de la psychanalyse. Le discours psychanalytique propose un modèle opposé à ces savoirs scientifiques modernes qui s'imposent dans le champ de l'éducation, qui bercent les éducateurs dans l'illusion d'une possible «gestion » du lien et d'une connaissance sans faille de ce qui constitue l'altérité et fait donc différence, en permettant ainsi sa maîtrise, pire son adaptation. Les critiques que Mannoni adressaient aux institutions éducatives dans les années 70 sont toujours de grande actualité (MANNONI, 2008). L'enjeu alors d'une psychanalyse qui se veut présente aux défis du champ social est de chercher et trouver comment permettre aux enseignants en formation d'accepter de travailler sur le fil de ce savoir qui ne se sait pas, et de ce malaise constitutif du lien à l'autre. Malaise et non mal-être!

C'est à partir de ces analyses que nous avons construit un dispositif de formation (PIRONE; WEBER, 2018) permettant aux enseignants, et plus largement aux professionnels des métiers de l'humain, de mettre au travail la question du rapport au savoir, la vérité du désir et ses enjeux de transmission, la relation aux élèves, et leur rapport aux discours pédagogiques et sociétaux. L'objectif est de pouvoir faire de la place, faire $d u$ vide, et au non-savoir pour pouvoir laisser place à la parole. Il s'agit pour nous de créer un lieu permettant aux professionnels de ne pas simplement « gérer» la relation à l'autre, autre-élève dans le cas des enseignants, ou bien autre-stagiaire dans le cas des conseillers pédagogiques qui forment les futurs enseignants au Luxembourg, mais de trouver comment être présent à la rencontre de l'autre. Cette opération implique la nécessaire prise en compte des impossibles qui cadrent notre existence et qui sont actuellement évités notamment par les discours techniques centrés sur les besoins, n'autorisant pas le sujet à écouter et à parler, à entendre un bout de la vérité qui parle en lui et en l'autre.

Ce que nous proposons est un dispositif d'analyse des pratiques professionnelles orienté par la psychanalyse, où les participants partagent dans l'espace du groupe des situations professionnelles qu'ils ont vécues et qui leur paraissent énigmatique, ou bien qui les mettent en souffrance, et sur lesquelles ils désirent réfléchir. L'enjeu est de permettre que la parole puisse circuler à nouveau.

Comme c'est le cas pour beaucoup de nos collègues qui animent des groupes d'analyse des pratiques professionnelles en France à partir d'une orientation psychanalytique, ${ }^{3}$ chaque séance est scandée par différentes phases, qui peuvent légèrement différer selon l'approche choisie pour la conduite du dispositif. Ce qui change essentiellement entre les différents formats, c'est le sens attribué à chacune de ces phases, le type d'analyse, sans oublier le style d'animation. Toutes ces différences dépendent du rapport singulier que chacun de nous a tissé avec la psychanalyse et des références que chacun a choisies tout au long de sa formation psychanalytique. Nous aimons rappeler que c'est notre façon de concevoir le sujet de l'inconscient qui détermine le style de la ren-

3 Nous renvoyons le lecteur au chapitre 11 de l'ouvrage de Francis Imbert, « Le groupe Balint dans le champ pédagogique » (IMBERT, 1997) et à l'ouvrage co-dirigé par Claudine Blanchard-Laville et Dominique Fablet (2001), Sources théoriques et techniques de l'analyse des pratiques professionnelles. Pour un panorama des différents usages actuels de ce dispositif, le lecteur peut consulter l'article co-écrit par Arnaud Dubois, Claudine Blanchard-Laville, et Sophie Lerner (2017), « Groupes d'analyse des pratiques pour enseignants. Mise en perspective de trois dispositifs inspirés du "groupe Balint" ». Dans le champ du travail socio-éducatif, le lecteur peut se référer à l'ouvrage de Joseph Rouzel (2007), La supervision d'équipes en travail social. 
contre. L'inconscient ne se réduit pas à un passé ou à un déjà-là ou à quelque-chose dont nous pourrions devenir conscient. C'est le fait que les mots nous manquent pour dire ce que nous sommes en tant que sujet de désir, en tant que parlêtre. L'écoute de l'analyste doit permettre un dire qui libère le sujet d'un savoir enfermant sur soi: "Pour que le sujet de l'inconscient garde sa forme spécifique d'existence, il est nécessaire que la psychanalyse déploie les conditions de son opérativité » (RECALCATI, 2010). ${ }^{4}$ Cette citation permet de surligner au fond le statut d'engagement nécessaire pour qu'une « rencontre » avec le sujet soit possible, donnant à l'analyste une fonction très active.

C'est donc à partir de ces quelques fondements que nous avons conçu ce dispositif d'analyse clinique des pratiques professionnelles, avec les références qui sont les nôtres. La forme que nous allons présenter est déterminée aussi par le fait que ces groupes se tiennent dans le cadre de nos formations universitaires. Chacun de nous anime ces groupes dans ces propres cursus universitaires, mais nous avons pu faire l'expérience de la co-animation pendant deux ans consécutifs au Luxembourg, dans le cadre de la formation des enseignants qui souhaitent devenir conseillers pédagogiques. Cette expérience nous a permis de partager et de co-construire une façon proche d'animer ces groupes, et de réfléchir ensemble aux défis éthiques et politiques d'une psychanalyse à l'université.

\section{Prendre le temps de: voir, comprendre, conclure}

Nous présenterons dans ce qui suit les différentes phases de ce dispositif en essayant d'en donner les enjeux.

Après avoir présenté le cadre du travail aux participants et rappelé les règles fondamentales du fonctionnement qui sont la confidentialité de ce qui se dit, l'abstention de

4 Traduit librement de l'italien, version numérique de l'ouvrage. tout jugement, et l'obligation pour chacun de présenter une situation, le travail commence. Les phases alors se répètent de la même façon pour chaque situation présentée.

Dans un premier temps, un participant du groupe présente une situation qu'il souhaite partager et qui le questionne. Une fois le récit terminé, le groupe pose des questions factuelles pour que des éléments soient précisés. Suit un deuxième temps, où l'étudiant qui a partagé la situation reste à l'écoute sans intervenir, pendant que les autres participants dégagent des pistes de lecture de la situation. Il reprend ensuite la parole pour revenir sur la situation initiale à partir des retours du groupe. Ce n'est qu'à la fin, dans un troisième temps, que les deux animateurs interviennent pour surligner certains éléments amenés tout au long des échanges et éventuellement les relier à des aspects théoriques, un choix qui est pour nous dicté par le fait que ces groupes sont intégrés dans des formations à l'université. C'est une occasion pour que des notions qui ont été abordées dans d'autres cours de la formation soient reprises dans la pratique, permettant ainsi que «quelque chose de la psychanalyse » soit transmis à l'université (FREUD, 1984). Au début de chaque séance, les participants qui ont présenté une situation la fois précédente, peuvent prendre la parole pour partager des éléments de réflexion, ou encore des questions restées en suspens.

La scansion, lors de ces différentes phases, a une fonction particulière et chaque temps est porté par des actions différentes, ayant des effets spécifiques. Mais pour toutes ses phases, il y a un facteur commun: le fait de prendre le temps, justement, pour créer un écart avec le temps accéléré de notre modernité (ROSA, 2010), de la réaction à la place de la réponse, du tout et tout de suite de l'impératif à la jouissance. Ces phases ${ }^{5}$ permettent de travailler sur différentes opérations qui sont au cœur de l'art d'enseigner et d'éduquer par les différences

5 Nous avons décrit ce dispositif dans un article publié en 2018 (PIRONE; WEBER, 2018). 
actions qui sont engagées. L’action de raconter, celle que Ricœur appelait la capacité de faire récit (RICOEUR, 2004), avec les opérations qui en découlent: rassembler; s'inclure dans la scène vécue ; s'adresser à un autre. L'action d'écouter, capacité d'écouter l'autre, et de s'entendre dire. L'action de partager: être avec les autres dans un groupe. Et pour finir, l'action de dire: faire retour à soi, s'entendre parler; chercher des pistes de lecture, non pas des interprétations, non pas des bribes d'analyse sauvage, mais des propositions de lecture ouvrant sur la suite symbolisant un point de désir au-delà.

Ces opérations représentent pour nous des temps logiques de subjectivation que nous pouvons comparer aux temps logiques que Lacan présente avec le fameux sophisme des prisonniers: l'instant de voir, le temps pour comprendre et le moment de conclure (LACAN, 1966).

Le premier temps, c'est le temps du récit. Nous demandons au participant qui veut mettre au travail une situation qu'il doit avoir vécue lui-même, de la raconter à la première personne, de la façon la plus détaillée possible. L'invitation faite aux participants de « raconter » une situation vise à soutenir ce travail de rassemblement et d'adresse aux autres, et permet de soutenir un déplacement vers une position active dans le dire. Souvent, dans une modalité peut-être aussi défensive, les participants choisissent de rapporter quelque chose qui se répète: nous leur proposons alors de choisir un moment précis dans la répétition. Quand, par exemple, le participant dit «ça se passe tous les jours », nous les invitons à faire un effort fictionnel, et à choisir un jour en particulier; ou bien encore à choisir un fragment, un événement précis, si la description prend le pas sur la narration. Nous invitons celui qui parle à dessiner les bords de la situation choisie par des marqueurs temporels et spatiaux. C'est pour nous un acte de ponctuation, et c'est déjà là, dès ces premiers instants, un travail de scansion permettant au sujet de s'entendre dire. Les participants présentent essentiellement des situations professionnelles qui les mettent en souffrance ou encore qu'ils ont vécues comme des échecs. Le travail d'élaboration doit permettre de dépasser une position de culpabilité, parfois paralysante, parfois point de jouissance, pour essayer de comprendre autrement ce qui a pu se jouer à un certain moment et dans un lieu précis. Un des points importants de ce type de travail est d'aider les praticiens à dépasser des formes de répétitions du même, où ça tourne en rond.

Mais ce que nous souhaitons pointer dans le fil de notre réflexion, c'est le fait que dans la répétition du même, les sujets donnent souvent l'impression de ne pas croire qu'ils ont d'autres choix, comme s'ils subissaient quelque chose d'inéluctable. D'où notre invitation à se situer, dans le sens d'expliciter l'événement qui a marqué une rupture en faisant un travail de nomination. Le but en est de ne pas laisser « tomber » le sujet dans un espace imaginaire atemporel et sans contours, et par conséquent sans possibilités de changement. Il s'agit de convoquer le sujet dans ce lieu de parole. Instant de voir.

Dans le deuxième temps, l'étudiant qui a raconté écoute, et ceux qui ont écouté peuvent parler. Écouter sans forcément dire quelque chose, c'est un apprentissage, une action. C'est une des capacités fondamentales dans les métiers où la relation à l'autre est centrale, capacité de laisser place à l'inconnu, à l'altérité, à l'énigme. Le groupe est alors invité à proposer des pistes de réflexion. Pas de jugement, c'est la règle du dispositif. Proposer activement, signifie ouvrir des perspectives et non pas réécrire une histoire d'une autre façon. Être tourné toujours vers l'avenir, c'est un enjeu éthique. Ce deuxième temps se termine avec la prise de parole du participant qui a partagé la situation et qui intervient suite aux pistes dégagées par les autres étudiants. Temps de comprendre.

Suit le troisième temps, les animateurs, qui ont jusque-là écouté et accompagné la scansion entre les différentes phases, prennent alors la parole pour la restituer au groupe. Il s'agit de 
re-dire, dans le sens de refaire un tour dans les échanges, de pointer des signifiants, surligner ce qui a été dit avec d'autres mots. Il ne s'agit pas d'interpréter, et non plus de donner des significations, d'expliquer, ou encore d'indiquer comment agir. Surligner signifie pointer les enjeux, et leur complexité. Moment de conclure, temps d'ouverture.

Il s'agit dans ce type de dispositif de parier sur le sujet à-venir. À partir de ce travail sur une rencontre avec l'imprévu et sur l'expérience du trou dans le savoir, ce qu'on vise, c'est que chacun découvre sa part de responsabilité par rapport à l'événement vécu. Une démarche qui va à l'encontre des processus de victimisation et de déresponsabilisation subjective produits par les nouveaux discours. C'est à partir de cette écoute que nous accueillons les situations amenées par les enseignants. Comme par exemple, quand un enseignant en formation pour devenir conseiller pédagogique, raconte à une séance du groupe d'analyse des pratiques professionnelles les difficultés qu'il rencontre avec le stagiaire qu'il accompagne, et qui est donc en formation pour devenir enseignant à son tour. Ces difficultés se répètent: il devrait y en avoir seulement un qui sait, le futur conseiller pédagogique, mais l'autre n'en veut pas de ce savoir, il sait aussi. Nous encourageons l'étudiant à choisir un moment en particulier dans cette série qu'il raconte: il décide alors de mettre au travail un moment d'échange entre lui et son stagiaire, après que le stagiaire a tenu une leçon devant la classe, c'est le moment dudit debriefing. En respectant les nouvelles injonctions à l'autonomie, qui suivent leur cours en parallèle avec celles sur la bienveillance, l'accompagnateur essaye par tous les moyens de faire réfléchir son stagiaire sur certaines situations de sa leçon, il ne (le) lâche pas. Il faut qu'il trouve « de façon autonome » ses erreurs, et plus précisément ceux que l'accompagnateur a repérées. Joli exemple des paradoxes éducatifs contemporains! Sauf que le stagiaire aussi se positionnait comme quelqu'un qui sait comment faire, et ces er- reurs lui ne les voyait pas du tout comme telles. Ses arguments ne convainquent pas le futur conseiller pédagogique. Les voilà alors dans une impasse, assujetti à un discours érigé pour l'un en discours de maître et pour l'autre en discours universitaire, l'un contre l'autre, savoir contre savoir, dans une forme spéculaire. Sans subjectivation et distance critique, on risque de ne plus tenir sa place, place de passeur, et de bloquer toute transmission. Même un discours comme celui de l'autonomie peut finir par laisser l'élève ou le stagiaire abandonné à soi-même, c'est-à-dire à sa jouissance. Tout le travail du dispositif d'analyse clinique des situations professionnelles consiste alors dans ce cas, à essayer de faire entendre cette position de maîtrise, de savoir-pouvoir, pour qu'une rencontre à l'avenir avec les futurs stagiaires soit possible.

Ces positions paradoxales du sujet tiraillé entre les discours éducatifs actuels et ses propres jouissances émergent très souvent aussi dans les situations que les enseignants amènent autour des questions touchant à l'inclusion scolaire. Voici un exemple parmi d'autres: une enseignante nous raconte sa difficulté à mener dans sa classe un travail d'inclusion d'un élève diagnostiqué à haut potentiel. Il présente, selon les dires de l'enseignante, des comportements de plus en plus dérangeants en classe, et il a de nombreux suivis à l'extérieur de l'école. Ce qui pose problème à l'enseignante, c'est son indiscipline et le traitement différent dont il bénéficie par rapport aux autres enfants. Le groupe des étudiants que nous accompagnons s'arrête longuement sur ce point, sur la difficulté de cette enseignante de ne pas savoir comment expliquer aux autres élèves pourquoi elle traite différemment cet enfant en particulier. Difficulté probablement à dire l'impossible qui se joue dans cette soi-disant différence, difficulté à lier par la parole les affects de cette rencontre avec l'altérité. L'enjeu du travail a été de permettre à l'étudiante de pouvoir exprimer son désir et d'entendre les défis qui se posent face à cette altérité, point que les élèves de la 
classe où elle travaille n'avait pas manqué de questionner.

\section{S'engager, rencontrer}

Le travail sur des situations transférentielles complexes permet d'inter-peller les enseignants en formation pour qu'ils puissent trouver comment faire avec les interdits fondamentaux, les impossibles, et pour qu'ils se déplacent et s'ouvrent au désir. En se rendant compte des transferts, des dépendances quand on met l'Autre à une place de sujet supposé savoir ou de sujet supposé non savoir, les étudiants s'entre-aident pour sortir en commun d'une impasse. Ce travail psychanalytique constitue un défi et un engagement éthique pour tous ceux qui y participent. « Le statut de l'inconscient [...] est éthique », affirmait Lacan (1973, p. 34), et nous en sommes responsables: « là où c'était, le je dois advenir ». Voilà une des raisons qui fait que l'être humain résiste à ce travail analytique et préfère se laisser moraliser, voire sadiser, par des soi-disant certitudes. Se confronter au désir et au discours de l'Autre aide les interlocuteurs à se positionner comme sujet, à sortir de leurs enfermements et idéologies. La rencontre permet de sortir de ses propres souffrances du langage, de s'arracher à cette souffrance en passant par la parole. $\mathrm{Ou}$ vert aux retours de l'autre nous finissons par mieux comprendre notre propre jouissance en jeu dans les échanges.

Le sujet ne se retrouve que là où l'être parlant fait le pari du sujet, là où il ose être à l'écoute de sa parole et de son corps. Ceci constitue un défi singulier, au cœur de la tâche de l'éducateur. En tant qu'être parlant adulte et professionnel, nous sommes des inter-locuteurs, ceux qui aident l'autre à s'exprimer, à s'expérimenter, à oser le pari de "dia-loguer », afin qu'un sujet de désir puisse émerger et poursuivre au-delà. Il s'agit finalement de créer un lien entre sujets qui assument leur responsabilité par rapport aux discours, aux pratiques et à leurs actes. En tant qu'analystes qui cadrent un tel travail nous nous engageons avec notre désir. C'est ainsi que nous suscitons le transfert des participants. C'est à travers la rencontre avec l'analyste et les Autres que la demande et en fin de compte le désir des étudiants sont suscités. La dynamique aide les participants à "dé-couvrir » les trous, à s'approcher du vide originaire, à se rendre compte finalement de la pulsion de mort (WEBER; VOYNOVA, 2020) qui déstabilise tout système, tout semblant de Grand Autre. L'assumer, c'est une des conditions pour risquer vivre des conflits et en débattre.

Ce type de travail joint éthique et politique, et en effet, la démocratie se construit sur du vide, de l'incertitude (LEFORT, 1986, p. 29). À nous d'inventer des parcours de vie et d'éducation, à condition d'accepter de faire avec l'impossible, en passant d'un discours à l'autre, comme nous le propose Lacan.

\section{Bibliographie}

ARENDT, H. La condition de l'homme moderne. Paris: Calman-Lévy, 1983.

BAUMAN, Z. La vita in frammenti. La morale senza etica del nostro tempo. Roma: Castelvecchi, 2018.

BLANCHARD-LAVILLE, C.; FABLET, D. (éd.) Sources théoriques et techniques de l'analyse des pratiques professionnelles. Paris: L'Harmattan, 2001.

CIFALI, M. Le lien éducatif: contre-jour psychanalytique. Paris: PUF, 1994.

DERRIDA, J. Marge de la philosophie. Paris: Les Éditions de Minuit, 1972.

DOUVILLE, O. Pour introduire l'idée d'une mélancolisation du lien social. Cliniques méditérranéennes, n. 63, p. 239-262, 2001.

DUBOIS, A.; BLANCHARD-LAVILLE, C.; LERNER, S. Groupes d'analyse des pratiques pour enseignants. Mise en perspective de trois dispositifs inspirés du "groupe Balint". Revue de Psychothérapie Psychanalytique de Groupe, n. 1, p. 115-130, 2017.

FREUD, S. Doit-on enseigner la psychanalyse à l'Université ? Dans Résultats, idées, problèmes I. Paris: PUF, 1984. p. 239-242.

FREUD, S. Malaise dans la civilisation. Paris: Payot \& Rivages, 2010. 
HAN, B.-C. La société de la fatigue. Strasbourg: Circé, 2014.

HAN, B.-C. Psychopolitique. Le néolibéralisme et les nouvelles techniques de pouvoir. Strasbourg: Circé, 2016.

IMBERT, F. L'inconscient dans la classe. Paris: ESF éditeur, 1997.

LACAN, J. Le temps logique et l'assertion de certitude anticipée. Un nouveau sophisme. Dans Écrits I. Paris: Éditions du Seuil, 1966. p. 195-211.

LACAN, J. Le Séminaire, Livre XI, Les quatre concepts fondamentaux de la psychanalyse. Paris: Seuil, 1973.

LACAN, J. Du discours psychanalytique. Dans CONTRI, G. B. (dir.). Lacan in Italia, Lacan en Italie, 1953-1978. Milan: La Salamandra, 1978. p. 26-39. Disponível em: http://societaamicidelpensiero.it/ wp-content/uploads/LACAN_ITALIA.pdf. Acesso em: 10 out. 2020.

LACAN, J. Je parle aux murs. Paris: Éditions du Seuil, 2011.

LEFORT, C. Essais sur le politique: XIXe et XXe siècles. Paris: Seuil, 1986.

MANNONI, M. Éducation impossible. Paris: Édi- tions du Seuil, 2008.

PIRONE, I.; TISSOT, P. L'inclusion scolaire, un enjeu de langage? Psychologie clinique, n. 50, p. 107116, 2020.

PIRONE, I.; WEBER J.-M. Comment être juste dans l'acte d'enseignement? Une question pour le sujet au-delà d'une compétence professionnelle. Spirale

- Revue de recherches en éducation, n. 61, p. 53-68, jan. 2018.

RECALCATI, M. L'uomo senza inconscio. Figure della nuova clinica psicoanalitica. Milano: Raffaello Cortina Editore, 2010. Version en ligne Kindle.

RICCEUR, P. Parcours de la reconnaissance. Paris: Éditions Stock, 2004.

ROSA, H. Accélération. Une critique sociale du temps. Paris: Éditions La Découverte, 2010.

ROUZEL, J. La supervision d'équipes en travail social. Paris : Dunod, 2007.

WEBER, J.-M.; VOYNOVA, R. Le décrochage scolaire: un rapport au savoir et la pulsion de mort. Montpellier: Champ social, 2020.

Recebido em : 30/08/2020

Aprovado em : $14 / 12 / 2020$ 